\title{
Migrations au Moyen-Orient
}

Hana JABER et France MÉTRAL (dir.), Monde en mouvements. Migrants et migrations au Moyen-Orient au tournant du XxI siècle, Beyrouth, Institut français du Proche-Orient (IFPO), 2005, 408 p.Françoise de BEL AIR, Migration et politique au Moyen-Orient, Beyrouth, IFPO, 2006, 218 p.

Delphine Pagès-El Karoui

\section{(2) OpenEdition}

1 Journals

\section{Édition électronique}

URL : http://journals.openedition.org/transcontinentales/919

DOI : 10.4000/transcontinentales.919

ISBN : 978-2-7351-1561-7

ISSN : $1775-397 X$

\section{Éditeur}

Editions de la maison des sciences de l'homme

\section{Édition imprimée}

Date de publication : 30 juin 2008

Pagination : 147-150

ISBN : 978-2-200-92511-6

ISSN : 1950-1684

\section{Référence électronique}

Delphine Pagès-El Karoui, « Migrations au Moyen-Orient », Transcontinentales [En ligne], 6 | 2008,

document 10, mis en ligne le 06 avril 2011, consulté le 22 septembre 2020. URL : http://

journals.openedition.org/transcontinentales/919; DOI : https://doi.org/10.4000/transcontinentales. 919 
Migrations au Moyen-Orient

Hana JABER et France MÉtral (dir.),

Monde en mouvements. Migrants

et migrations au Moyen-Orient au tournant

du XXI siècle, Beyrouth, Institut français

du Proche-Orient (IFPO), 2005, 408 p.

Françoise de BEL AIR,

Migration et politique au Moyen-Orient, Beyrouth, IFPO, 2006, 218 p.

Le Moyen-Orient a une position originale dans les flux de population internationaux. Premier récepteur de migrants venus du monde en développement, il est également émetteur de flux migratoires et zone de transit. Les pays du Golfe, grâce à la manne pétrolière, sont devenus un pôle attractif pour une main-d'ouvre qualifiée et non qualifiée, arabe comme asiatique. Dans le même temps, les bouleversements géopolitiques qui ont affecté la région (conflits israélo-arabes, guerre civile libanaise, guerres du Golfe...) ont provoqué d'importantes vagues de réfugiés. À un an d'intervalle, deux ouvrages ont été publiés par l'Institut français du Proche-Orient (IFPO) sur cette thématique. Le premier, Mondes en mouvements, coordonné par H. Jaber et F. Métral, rend compte de la diversité et de la complexité des situations migratoires depuis le début des années 1990, que les auteurs présentent comme un tournant majeur. D'abord, parce que la guerre du Golfe a suscité une réorientation massive des flux : retours forcés de travailleurs dans le Golfe (Égyptiens en Irak, JordanoPalestiniens au Koweït...), réfugiés irakiens en Turquie et en Iran. Ensuite, parce que les années 1990 symbolisent pour les pays du Moyen-Orient l'entrée de plain-pied dans la mondialisation, accélérant la circulation des hommes, des marchandises et des capitaux.

Si la diversité des situations migratoires est parfaitement illustrée par la quinzaine de contributions, toutes de bonne tenue scientifique et portant sur des champs très variés, on regrette que l'introduction n'apporte pas suffisamment de fondements théoriques pour l'esquisse d'une réflexion plus large, transcendant la multitude d'études de cas, aussi intéressantes soient-elles. Bien que les nombreux renvois d'un texte à l'autre attestent bien d'un travail collectif, la dimension comparative est peu présente. L'approche de la complexité semble se réduire à remettre en cause la traditionnelle distinction entre migrations économiques et migrations forcées. Le second ouvrage, Migration et politique, dirigé par F. de Bel Air, s'inscrit dans le prolongement du premier avec, comme son titre l'indique, une entrée thématique plus resserrée et davantage problématisée dans l'introduction. L'éventail géographique des huit contributions est lui aussi plus restreint, puisqu'il se limite à la Jordanie, au Liban et à la question des réfugiés palestiniens.

Parmi la multitude de thématiques qui s'enchevêtrent dans les deux ouvrages, retenons, pour simplifier, deux grands axes : une approche par le haut (l'État et les politiques migratoires) et une approche par le bas, qui est centrée sur les migrants. Selon ce dernier point de vue, la mobilité apparaît comme une compétence, permettant d'accroître ses revenus mais aussi son statut social et/ou de fuir un pays où règne l'insécurité. À l'heure où les États occidentaux contrôlent de plus en plus étroitement leurs frontières et où, après le 11 septembre, une méfiance accrue vise les ressortissants du Moyen-Orient, la mobilité est perçue comme un atout indispensable. D'où les nombreuses stratégies d'acquisition de nationalités (et notamment de la nationalité canadienne) qui offrent la facilité de circuler plus librement dans le monde globalisé. F. Adelkhah évoque même pour les Iraniens une émigration 
virtuelle, décrivant des pratiques de préparation en vue d'un hypothétique départ qui risque bien de ne jamais se concrétiser (cours d'anglais, passage du TOEFL, obtention d'un visa, participation à la loterie des green cards). L'expérience migratoire, lorsqu'elle est temporaire, est dans bien des cas l'occasion pour des jeunes gens de s'émanciper de la tutelle familiale: leur épargne constituée pendant l'expatriation leur permettra à leur retour de se marier et d'établir un foyer indépendant de la famille. L'émigration contribue en ce sens à l'individualisation de la société, via la promotion de la famille nucléaire. Pour autant, le savoir-faire migratoire repose aussi, comme cela a été souvent décrit, sur des réseaux de solidarité qu'ils soient familiaux, communautaires ou locaux (Doraï). Quelques contributions éclairent des domaines moins explorés : la circulation des migrants qualifiés au sein des firmes transnationales retient l'attention d'E. Longuenesse; une approche par le genre des migrations se développe avec l'essor de la mobilité des femmes seules - domestiques philippines (Jaber) ou jeunes femmes russes qui se livrent au commerce à la valise ou à la prostitution à Dubaï (Meiering).

Le deuxième axe traite des liens entre politique et migration, thème qui est approfondi dans l'ouvrage de F. de Bel Air. Le constat est identique : contrairement à ce qui a été un temps annoncé du fait de la mondialisation et de l'essor des migrations internationales, les États restent très puissants dans le contrôle des flux de population, même si les politiques varient d'un pays à l'autre. Certains font tout pour entraver le départ de leurs concitoyens, comme l'Irak dans les années 1990 qui imposa des taxes de sortie très élevées et multiplia les mesures d'intimidation (Chatelard); d'autres, comme les Philippines ou l'Égypte à partir des années 1970, ont encouragé l'exportation de leur main-d'œuvre.

Quant à l'immigration, elle est étroitement contrôlée partout. Avec la kafala, le contrôle des migrations est délégué aux ressortissants nationaux. Ce système d'abord mis en place dans les pays pétroliers, puis étendu à d'autres États de la région, impose au migrant d'avoir recours à un intermédiaire, le kafil, garant de sa présence sur le territoire et qui, contre rétribution, assure toutes les démarches administratives. La kafala instaure une segmentation du marché du travail, car il est difficile pour la main-d'œuvre non qualifiée de changer de type d'emploi. Peu compatible avec la libéralisation économique, la kafala est partiellement démantelée dans les Émirats arabes unis, notamment à l'intérieur des zones franches qui se multiplient (Rycx). Les nationaux, cependant, verraient d'un mauvais œil la disparition totale du système qui leur supprimerait une rente non négligeable.

Après la crise économique de 1986 et la montée du chômage des nationaux, les politiques de contrôle des flux se sont renforcées et l'accès de la main-d'œuvre étrangère au marché du travail a été restreint aux emplois les moins qualifiés, l'objectif global étant de limiter la dépendance à l'égard des immigrés (aux Émirats arabes unis, plus de $80 \%$ de la population active est étrangère). En Jordanie, seuls les services domestiques, la construction et l'agriculture sont restés ouverts aux non-Jordaniens. Partout, le statut des étrangers est très précaire: généralement, ils ne bénéficient ni de droits sociaux, ni de couverture médicale, et se voient interdire l'adhésion à des syndicats. De plus, la majorité des pays arabes ne sont pas signataires de la convention de Genève. Ils laissent le soin 
au Haut-Commissariat des Nations unies pour les réfugiés (HCR) d'accorder ou non le statut de réfugiés, à condition que ceux-ci, une fois reconnus, soient réinstallés dans un pays tiers (Europe, ÉtatsUnis, Canada). La grande précarité des immigrés au Moyen-Orient, la montée de la xénophobie et l'instrumentalisation de l'immigration par les pouvoirs publics ont créé de fortes tensions qui ont mis à mal le mythe de l'unité panarabe. $\mathrm{Si}$, dans un premier temps, la venue de travailleurs arabes a été encouragée (absence de visa, embauche préférentielle), on assiste depuis les années 1990 à son remplacement par une main-d'œuvre asiatique jugée moins chère et plus docile. Stigmatisés comme des migrants illégaux, constamment menacés d'expulsions, marginalisés dans un pays arabe, les ouvriers égyptiens en Jordanie par exemple ont fait l'expérience douloureuse de l'altérité dans un contexte culturel pourtant censé être proche. Leur exploitation leur est apparue d'autant plus choquante qu'ils s'imaginaient faire partie d'un même ensemble. Cette situation les a conduits, en réaction, à reformuler leur identité égyptienne (Lafargue).

Un autre questionnement s'impose alors : comment les migrants participentils à de nouvelles formes de construction nationale? La mondialisation et l'essor des migrations internationales ont ébranlé le modèle de l'État-nation et nécessitent de repenser les concepts de nation, de citoyenneté et de territoire national. Le projet d'un futur État palestinien qui encouragerait le retour de réfugiés illustre bien ces nouveaux enjeux : comment concilier la très grande flexibilité des pratiques transnationales des réfugiés palestiniens avec l'inflexibilité des États? S. Hanafi propose d'imaginer un nouveau modèle « d'État-nation extra-territorial» qui distinguerait la citoyenneté de la nationalité (les droits et devoirs de ceux qui résident en Palestine ne seraient pas fonction de leur nationalité, tandis que ceux qui vivent à l'étranger pourraient disposer de la citoyenneté et intervenir dans certains domaines de la vie politique).

Au-delà du cas particulier palestinien, les États au Moyen-Orient se caractérisent par l'importance des rentes dans l'économie (hydrocarbures, aide internationale, transfert d'épargne des émigrés...), avec une légitimité étatique qui repose historiquement sur la redistribution des rentes et qui justifie l'absence de participation politique des citoyens par le faible recours aux prélèvements directs. L'exercice du politique se réduit donc à la redistribution de la rente selon une logique clientéliste et cantonne le citoyen dans son rôle de consommateur. Le désengagement progressif des États des logiques de redistribution, imposé par les politiques d'ajustement structurel, a accentué le divorce grandissant entre l'État et sa population, réactivant des appartenances infranationales, d'ordre tribal par exemple, ou supranationales, avec le succès d'idéologies pan-islamistes, transcendant les frontières. Parallèlement, la fragmentation accrue des sociétés conduit à autant de divergences sur la définition de l'identité nationale.

Dans Migration et politique, les politiques migratoires sont examinées en privilégiant la méthode de l'analyse institutionnelle, qui permet de décrypter les enjeux de la stabilité et de la souveraineté nationales, déclinés autour de deux thèmes majeurs. La question du nombre d'abord, liée à la définition de la population mais aussi du territoire national, qui est cruciale pour de nombreux pays et conduit à l'instrumentalisation des 
migrations. Au Liban, de virulents débats ont agité l'opinion publique au sujet de l'attribution de la nationalité libanaise aux descendants des émigrés et de l'éventuel droit de vote des expatriés (Jaulin). L'enjeu est clair : en préférant le droit du sang à celui du sol, les chrétiens souhaiteraient réintégrer dans la vie politique libanaise les membres de la diaspora, chrétiens pour la moitié, alors que la démographie est depuis plusieurs décennies en leur défaveur. Cette vision a été fortement soutenue par l'Église maronite, qui reste très active dans le processus de construction nationale libanais, tout en se transformant en une institution diasporique qui multiplie les nouvelles implantations à l'étranger et cherche constamment à resserrer les liens entre les communautés maronites expatriées et l'Église-mère du Liban (Tabar).

La question du contrat social est, elle aussi, récurrente. C'est en ce sens qu'est envisagée l'offre de services domestiques comme élément de confort matériel, en échange de l'allégeance politique des bénéficiaires. Aux Émirats arabes unis, on dénombre davantage de domestiques étrangères - ce sont surtout des jeunes femmes asiatiques - que de nationaux! Ces domestiques sont soumis au pouvoir discrétionnaire des employeurs, les inspecteurs du travail ayant l'interdiction de pénétrer à l'intérieur de la cellule familiale, traditionnel sanctuaire de la vie privée. Cette privatisation de la vie sociale (garde de jeunes enfants, entretien de la maison, soins aux personnes dépendantes...) permet à l'État de promouvoir une classe moyenne moderne, fondée sur la famille nucléaire et l'affaiblissement des liens de solidarité familiale, tout en renforçant le conservatisme et les structures patriarcales de la société. En effet, la maison reste le domaine féminin par excellence mais se transforme en une sphère intermédiaire entre le privé et le public: la maîtresse de maison endosse le rôle d'employeur professionnel, sorte de manager disposant d'un contrôle total sur les domestiques étrangères. Elle acquiert ainsi un nouveau statut social et un sentiment de supériorité qui conforte son appartenance nationale. Ainsi la ségrégation à l'emploi selon les conditions de travail permet à la distinction entre citoyens et non-citoyens de s'opérer.

Ces deux ouvrages, par la richesse des thèmes abordés et l'actualisation des connaissances qu'ils fournissent, seront très utiles pour tous ceux qui s'intéressent aux migrations internationales et au Moyen-Orient. Ils appellent une réflexion plus large sur cette question qui commence à être bien défrichée, tandis que certains champs mériteraient une plus grande attention, tels l'Europe comme «nouvelle» destination des migrants moyen-orientaux ou encore l'externalisation des politiques migratoires de l'Union européenne.

Delphine Pagès-El Karoui, Institut national des langues et civilisations orientales, centre de recherches MoyenOrient et Méditerranée (INALCO CERMOM)/ Migrinter

\section{Atlas du Liban}

\section{Éric VerdeIL, Ghaleb FaOur}

et Sébastien VeLut,

Atlas du Liban. Territoires et société, Beyrouth, Institut français du ProcheOrient-CNRS Liban, 2007, 207 p.

L'Atlas du Liban qui vient de paraître propose une lecture originale de l'espace libanais contemporain (seules quelques cartes historiques sont présentées pour mettre en perspective la construction nationale libanaise). L'originalité de cet atlas repose sur la multiplicité des sources employées et leur mise en 